\title{
Gaze and Gesture Based Object Manipulation in Virtual Worlds
}

\author{
Dana Slambekova \\ Rochester Institute of \\ Technology \\ Rochester, NY, USA \\ dxs4659@rit.edu
}

\author{
Reynold Bailey \\ Rochester Institute of \\ Technology \\ Rochester, NY, USA \\ rjb@cs.rit.edu
}

\author{
Joe Geigel \\ Rochester Institute of \\ Technology \\ Rochester, NY, USA \\ jmg@cs.rit.edu
}

\begin{abstract}
In this work we present a framework for enabling the use of both eye gaze and hand gestures for interaction within a $3 \mathrm{D}$ virtual world. We define a set of natural interaction mechanisms for manipulation of objects within the 3D space and describe a prototype implementation based on Second Life that allows these mechanisms to be used in that world. We also explore how these mechanisms can be extended to other spatial tasks such as camera positioning and motion.
\end{abstract}

\section{Categories and Subject Descriptors}

I.3.7 [Computer Graphics]: Three-Dimensional Graphics and Realism - Virtual reality

\section{Keywords}

virtual reality;gesture recognition;eye tracking; Kinect

\section{INTRODUCTION}

Motivated by interfaces depicted in films such as Minority Report, much attention has been placed on the use of spatial gestures for interactions in $3 \mathrm{D}$ virtual spaces $[1,2]$. Research on natural gaze behavior and hand-eye coordination during object manipulation suggests that the use of gaze data, in addition to gesture based methods, may increase the effectiveness of performing these tasks in a virtual space.

\section{ARCHITECTURE \& INTERACTION}

The system (Figure 1) provides two paths for user interaction: detection and recognition of $3 \mathrm{D}$ gestures and the tracking the eye gaze of the user. Together, these signals are used to trigger actions from a set of predefined mappings: from gesture / gaze to controllable events within the virtual space. These events are then realized within a particular virtual world environment using the mechanisms available in that environment.

We combine a "look at" mechanism for choosing objects with a handle bar metaphor[2] for object manipulation. Hand state is used as a trigger for selection and deselection of objects while eye gaze data is used to determine the object on which to apply the selection. Once selected, objects can be translated, rotated, and scaled by use of intuitive 3D gestures as shown in Figure 2. For translation, eye gaze is also used to locate a target position for the translated object.

Copyright is held by the author/owner(s).

VRST'12, December 10-12, 2012, Toronto, Ontario, Canada.

ACM 978-1-4503-1469-5/12/12.

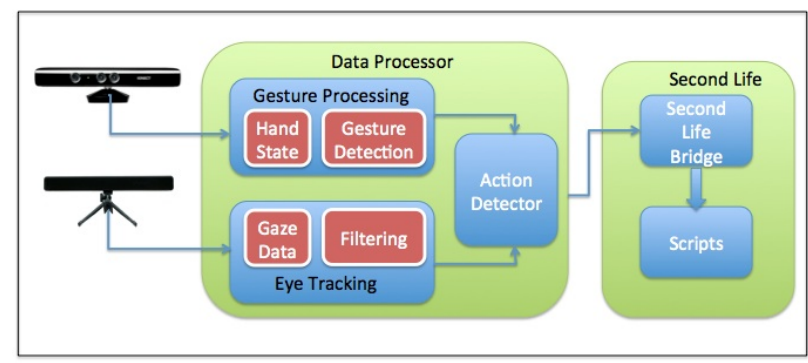

Figure 1: System Architecture

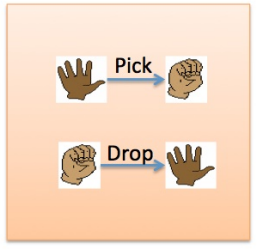

(a) translation

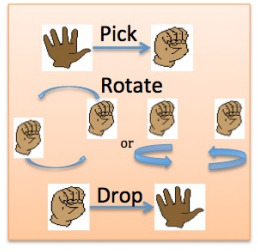

(b) rotation

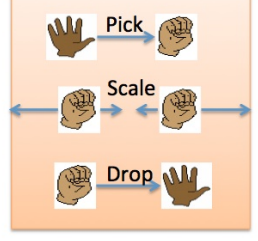

(c) scaling
Figure 2: Interaction Mechanisms

We have implemented a prototype of the system using a Microsoft Kinect sensor for gesture capture, a Mirametrix eye tracker to detect eye gaze and Second Life as the target virtual world platform. The prototype serves as a proof of concept and a framework for testing other kinds of interaction based on gesture and gaze (e.g. camera motion).

\section{REFERENCES}

[1] G. Hackenberg, R. McCall, and W. Broll. Lightweight palm and finger tracking for real-time $3 \mathrm{~d}$ gesture control. In Virtual Reality Conference (VR), 2011 IEEE, pages $19-26$, march 2011.

[2] P. Song, W. B. Goh, W. Hutama, C.-W. Fu, and X. Liu. A handle bar metaphor for virtual object manipulation with mid-air interaction. In Proceedings of the 2012 ACM annual conference on Human Factors in Computing Systems, CHI '12, pages 1297-1306, New York, NY, USA, 2012. ACM. 